Check for updates

Cite this: RSC Adv., 2018, 8, 26432

Received 30th June 2018

Accepted 11th July 2018

DOI: $10.1039 / \mathrm{c} 8 \mathrm{ra05600e}$

rsc.li/rsc-advances

\section{Robust microfluidic construction of hybrid microfibers based on konjac glucomannan and their drug release performance $\uparrow$}

\author{
Yongsheng $\mathrm{Ni}^{,}{ }^{a}$ Wanmei Lin, ${ }^{a}$ Ruo-Jun $\mathrm{Mu},{ }^{a}$ Chunhua $\mathrm{Wu},{ }^{a}$ Lin Wang, ${ }^{a}$ Dan $\mathrm{Wu},{ }^{a}$ \\ Su Chen (1D) ${ }^{* b}$ and Jie Pang*a
}

\begin{abstract}
The exploration of methods to produce a novel wound dressing with sustained drug release properties in ultrasmall scales is of great scientific and technological interest. Herein, we propose konjac glucomannan/ polyvinylidene fluoride (KGM/PVDF) hybrid microfibers having hydrophilic and hydrophobic segments based on microfluidic-oriented core-sheath composite microfibers, where the KGM/PVDF hybrid microfibers are wrapped in situ in $\mathrm{CH}_{3} \mathrm{OH}$. The morphology of KGM/PVDF microfibers is uniform, smooth, and crack-free. Enrofloxacin (Enro) is loaded onto the microfibers as a representative cargo to test their release performance. The KGM/PVDF/Enro microfibers show sustained drug release performance (13 days), excellent heat resistance, antibacterial activity and promotion of wound healing. This study is an avenue toward the microfluidic design of hydrophilic/hydrophobic hybrid microfibers as wound dressings, and it will guide the development of next-generation wound dressing.
\end{abstract}

\section{Introduction}

Designing high performance microfibers as wound dressings has drawn considerable attention because of their release behavior, ${ }^{1}$ healing function, ${ }^{2}$ biocompatibility, ${ }^{3}$ biodegradability, ${ }^{4}$ and sterilization. ${ }^{5}$ These microfibers have been used for wound hemostasis, ${ }^{6,7}$ wound repair, ${ }^{8,9}$ and as burn dressings. ${ }^{10}$ Recently, many approaches have been proposed for the construction of microfibers, and these approaches include polymer cold-drawing, ${ }^{11}$ self-assembly, ${ }^{12}$ electrospinning, ${ }^{13}$ and microfluidic spinning. ${ }^{14}$ Many reports have focused primarily on microfibers produced by electrospinning. For example, Xia's group reported uniform microfibers with high energy-transfer efficiency via electrospinning. ${ }^{15}$ Mulhaupt's group constructed attractive high-surface-area polyvinyl alcohol (PVA) microfibers by electrospinning. ${ }^{16}$ Lee's group built an angiogenic microfiber that directs blood vessel growth via binding electrostatically after electrospraying. ${ }^{17}$ Despite the fact that different types of microfibers have been developed, it is still challenging to create

${ }^{a}$ College of Food Science, Fujian Agriculture and Forestry University, Fuzhou 350002, China. E-mail: pang3721941@163.com; Fax: +86-25-83756316; Tel: +86-2583756316

${ }^{b}$ State Key Laboratory of Materials-Oriented Chemical Engineering, College of Chemical Engineering, Jiangsu Key Laboratory of Fine Chemicals and Functional Polymer Materials, Nanjing Tech University (former Nanjing University of Technology), Nanjing 210009, China. E-mail: chensu@njtech.edu.cn; Fax: +86-2583172258; Tel: $+86-25-83172258$

$\dagger$ Electronic supplementary information (ESI) available. See DOI: $10.1039 / \mathrm{c} 8 \mathrm{ra} 05600 \mathrm{e}$ new types of drug-loaded microfibers with sustained release performance.

Microfluidic spinning enables precise manipulation of fluids within microscale channels and thus, it has drawn considerable attention due to its simplified manipulation, environmentally friendly chemical processes, and flexible controllability. ${ }^{18-20}$ In particular, these microfibers are normally arranged in order arrays, that is, they significantly benefit the controlled movement of a cargo carrier by microfluidic spinning technology. ${ }^{21}$ In this aspect, in our previous study, we reported on the possibility of concisely constructing photonic crystal-loaded bead-shaped hybrid microfibers arrays ${ }^{22}$ and drug-loaded ${ }^{23}$ materials via microfluidic spinning. However, the design of highperformance functional microfibers is still challenging.

Herein, we report a simple and rapid strategy for the in situ preparation of konjac glucomannan/polyvinylidene fluoride (KGM/PVDF) hybrid microfibers having hydrophilic and hydrophobic segments via microfluidic spinning, and the microfibers can serve as a candidate for a new type of wound dressing. In this case, KGM is a natural polysaccharide consisting of hydrophilic glucose and mannose, allowing these microfibers to have high drug-load efficiencies, good biocompatibility, and good biodegradability. ${ }^{24-27}$ PVDF is a hydrophobic thermoplastic semi-crystalline polymer, ${ }^{28-30}$ which offers good tensile strength, easy processing and drug release properties. ${ }^{31}$ More importantly, enrofloxacin (Enro) was reported by Zhou's group ${ }^{32}$ to show 3 day sustained drug release performance when used as a wound dressing, and we have employed it as the target drug loaded on these hybrid microfibers during the production of robust bacterial infection wound dressings to 
validate the availability of our idea. This method demonstrates an available route for designing a brand new hybrid microfiber combining hydrophilic and hydrophobic segments; the hybrid microfibers exhibit excellent drug release performance and can be used as novel wound dressings.

\section{Experimental}

\subsection{Materials}

KGM was purchased from Shaotong Sanai Konjac Development Co. PVDF was purchased from Sigma-Aldrich (St. Louis, MO, USA). Enro were purchased from Sinopharm Chemical Reagent Co., Ltd (Shanghai, China).

\subsection{Preparation of different KGM/PVDF hybrid microfibers}

A microfluidic spinning machine (from Nanjing Janus New Materials limited company, JNS-MS-03) was used to construct the microfibers. First, 2 wt\% KGM solution was prepared in $100 \mathrm{~mL}$ purified water with stirring for $6 \mathrm{~h}$ at $400 \mathrm{rad} \mathrm{min}^{-1}$. A $25 \mathrm{wt} \%$ PVDF solution was prepared in $100 \mathrm{~mL}$ DMAC/ACE $(1: 1, \mathrm{v} / \mathrm{v})$ with a stirring rate of $500 \mathrm{rad} \mathrm{min}^{-1}$ until PVDF was completely dissolved. Next, the KGM/PVDF spinning foundation liquid was prepared by mixing $2 \mathrm{wt} \% \mathrm{KGM}$ solution and $25 \mathrm{wt} \%$ PVDF at weight ratios of $0: 25,1: 20,1: 15$ and $1: 10$. The spinning foundation liquid was treated with ultrasonication until a clear solution was obtained. The solution was loaded into a syringe $(20 \mathrm{~mL})$ with a $16 \mathrm{G}$ stainless steel needle. The microfiber spinning equipment consisted of two syringe pumps, a coaxial continuous T-shaped microfluidic platform, and a frame receiver. The core flow of the KGM/PVDF solution was pumped into the microdevice through a needle microchannel. The $\mathrm{CH}_{3} \mathrm{OH}$ sheath flow was injected into the region between the inner needle microchannel and the outer silicone rubber hose. KGM/PVDF was instantly encapsulated at the interface between the two flows because KGM is insoluble in methanol solution. ${ }^{33}$ The encapsulated KGM/PVDF microfibers were then extruded through an outlet channel and continuously collected with a frame receiver.

\subsection{Morphology of microfibers and antibacterial drug loading}

The morphology of the microfibers was observed with scanning electron microscopy (SEM) using the Hitachi S-4800 system. The formation defects measured the optimal weight ratios of KGM : PVDF. In addition, Enro was typically loaded into the core flow to form KGM/PVDF/Enro microfibers. Different concentrations of $\mathrm{KGM} / \mathrm{PVDF} /$ Enro spinning solutions were prepared by first dissolving the optimal weight ratio KGM/PVDF spinning foundation liquid based on the SEM results. Then, different weights of Enro were added to the KGM/PVDF spinning foundation liquid: $10 \%, 15 \%$, and $20 \%$ of PVDF (w/w) are denoted as E1, E2, E3, respectively; as controls, pure PVDF spinning liquid samples were also loaded with the same drug concentrations, and they were denoted as B1, B2, and B3, respectively. All solutions were degassed before microfluidic spinning.

\subsection{The characterization of hybrid microfibers}

FTIR spectra were obtained on a Nicolet-6700 spectrometer from Thermo Electron at room temperature. The purified samples were ground into powder with $\mathrm{KBr}$ and pressed into a pellet. We collected 32 scans with $4 \mathrm{~cm}^{-1}$ resolution. Thermal behaviors were analyzed with a synchronous thermal analyzer (STA449 F3) at $15 \mathrm{~K} \mathrm{~min}^{-1}$ from $50{ }^{\circ} \mathrm{C}$ to $575{ }^{\circ} \mathrm{C}$. For X-ray diffraction (XRD), we used an ARL X'TRA powder X-ray diffractometer with a $\mathrm{Cu}-\mathrm{K} \alpha$ radiation source $(\lambda=0.1542 \mathrm{~nm})$ at a scanning speed of $5 \mathrm{~min}^{-1}$ with a $2 \theta$ range of $5^{\circ}$ to $80^{\circ}$.

\subsection{Drug release in vitro}

The drug release behavior of the microfibers was investigated in $\mathrm{PBS}$ at $\mathrm{pH} 7.4 \pm 0.2$. We selected $25^{\circ} \mathrm{C}$ as the room temperature and $37{ }^{\circ} \mathrm{C}$ as the body temperature. To evaluate the maximum absorption wave of Enro, different Enro solutions were scanned from $200 \mathrm{~nm}$ to $400 \mathrm{~nm}$ using a UV-vis spectrophotometer (UV2600, Japan), and the amount of Enro was calculated from the calibration curve. The encapsulation efficiencies (EEs) and drug loading efficiencies (DLEs) of the microfibers were determined using the following equations: ${ }^{34}$

$$
\begin{gathered}
\operatorname{EEs}(\%)=\frac{m_{\mathrm{A}}}{m_{\mathrm{T}}} \times 100 \\
\operatorname{DLEs}(\%)=\frac{m_{\mathrm{A}}}{m_{\mathrm{M}}} \times 100
\end{gathered}
$$

here, $m_{\mathrm{A}}$ and $m_{\mathrm{T}}$ are the actual and theoretical Enro contents of the microfiber, respectively, and $m_{\mathrm{M}}$ is the weight of the dry microfibers.

The Enro-loaded microfibers $(80 \mathrm{mg})$ were incubated in $80 \mathrm{~mL}$ PBS at $25^{\circ} \mathrm{C}$ or $37^{\circ} \mathrm{C}$ with constant shaking at $60 \mathrm{rpm}$. An $8.0 \mathrm{~mL}$ aliquot was periodically removed to quantify drug release with UV-vis; the same amount of fresh medium was added to maintain a volume of $80 \mathrm{~mL}$. The results were presented in terms of cumulative release (CR, \%). All release studies were performed in triplicate, and the results were reported as the average values \pm S.D. CR was calculated according to the following equation: ${ }^{35}$

$$
\mathrm{CR}(\text { Enro })=\frac{80 C_{n}+\sum 8 C_{n-1}}{m_{\mathrm{s}}} \times 100 \%
$$

here, $C_{n}$ and $C_{n-1}$ are the concentrations of Enro in the release media at $n$ and $n-1$ times, respectively, $n$ is the number of sample time points, and $m_{\mathrm{s}}$ denotes the initial amount of Enroloaded.

\subsection{Differential scanning calorimetry (DSC)}

DSC was measured with a DSC200F3 instrument (Zetzsch, Germany) from $25{ }^{\circ} \mathrm{C}$ to $400{ }^{\circ} \mathrm{C}$ at a rate of $10{ }^{\circ} \mathrm{C} \mathrm{min}{ }^{-1}$ under

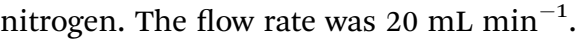

\subsection{Infrared imaging (IR imaging)}

IR images were obtained using a Thermo Scientific Nicolet iN10 infrared microscope equipped with a liquid nitrogen-cooled MCT detector (Thermo Electron Corporation, USA). IR 
microscopy data were collected using reflection mode, and IR spectra were recorded from 4000 to $400 \mathrm{~cm}^{-1}$. Analysis of the IR microscopy data was performed using the OMNIC Picta software (Thermo Electron Corporation, USA).

\subsection{Test of antibacterial activity}

The antibacterial activities of PVDF/Enro and KGM/PVDF/Enro microfibers were tested with the inhibition-zone method. ${ }^{36} S$. aureus and $E$. coli were cultivated in nutrient broth $(3 \mathrm{~g}$ beef extract, $10 \mathrm{~g}$ peptone, and $5 \mathrm{~g}$ sodium chloride per $1000 \mathrm{~mL}$ water). S. aureus and E. coli (approximately $10^{7}$ to $10^{8} \mathrm{CFU} \mathrm{mL}^{-1}$ ) were then evenly spread over LB agar plates. The resulting samples (circular sheet, $10 \mathrm{~mm}$ diameter) were placed on the LB agar plates, and the plates were incubated at $37^{\circ} \mathrm{C}$ for $24 \mathrm{~h}$. The antibacterial effects of the samples were assessed by comparing the size of the inhibition zones, as measured with calipers. ${ }^{37}$

\subsection{Wound dressing experiments}

The wound healing experiments were performed at Fu Zhou General Hospital and approved by the Ethics Committee of $\mathrm{Fu}$ Zhou General Hospital (approval no. 2017-1220). International ethical guidelines and the National Institutes of Health Guide concerning the Care and Use of Laboratory Animals were strictly followed. Mice were obtained from the Fuzhou Institute of Experimental Animals (Fujian, China). Eight-week-old male Sprague Dawley rats (250 g; $n=18$ ) were anesthetized and then, $1.2 \mathrm{~cm} \times 1.2 \mathrm{~cm}$ wounds were created on the dorsum of the mice under sterile conditions. ${ }^{38}$ The mice were randomly divided into three groups: (i) control group (gauze covered), (ii) PVDF/Enro microfibers covered, and (iii) KGM/PVDF/Enro hybrid microfibers covered. The mice were recovered from anesthesia and housed in separate cages. A gross view of the wound was seen at $0,3,7,10$, and 13 days after operation. The wound closure rates were calculated based on comparisons with the original area of the wound. At 3 and 7 days, the mice were sacrificed, and the wounds were excised including a $5 \mathrm{~mm}$ surrounding area of the intact tissue. The tissues were immersed in paraformaldehyde (4\%) for $24 \mathrm{~h}$ and then embedded in paraffin. After the paraffin was sectioned, hematoxylin-eosin (H\&E) staining was performed for histological analysis. $^{39}$

\section{Results and discussion}

\subsection{In situ preparation of microfibers}

We started the successful construction of hybrid microfibers with the use of hydrophilic KGM and hydrophobic PVDF as spinning precursors. For the production of drug-loaded microfibers, we employed the drug Enro to load into the hybrid microfibers, allowing the microfibers to be used as wound dressings (Fig. 1). In a typical run, KGM/PVDF hybrid microfibers were prepared by changing the weight ratios of KGM and PVDF $(0: 25,1: 20,1: 15$ and $1: 10)$. Then, the ordered microfiber arrays were observed by SEM. As seen in Fig. 2a, the surface of pure PVDF microfibers was flat and rough, and it had several fissures. However, these fissures reduced with

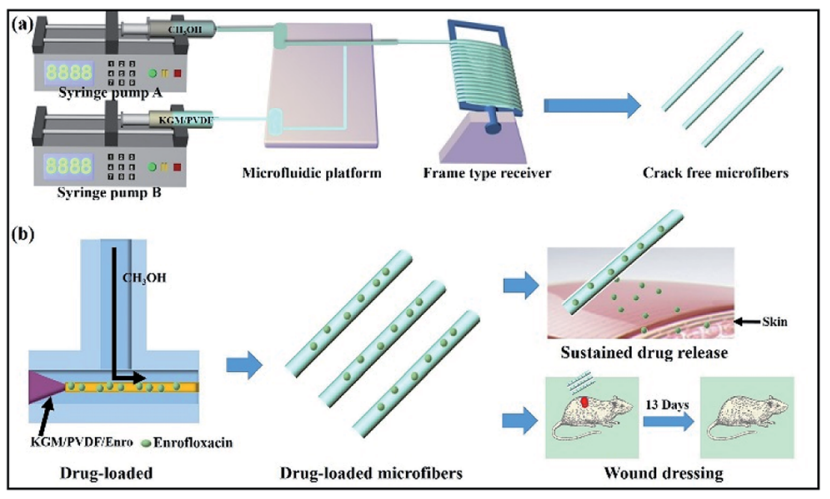

Fig. 1 (a) Schematic of the microfluidic preparation of KGM/PVDF crack-free hybrid microfibers. KGM/PVDF was chosen as the core flow, and the sheath flow was $\mathrm{CH}_{3} \mathrm{OH}$ for encapsulation in a T-shaped microfluidic platform. (b) Drug loading and release performance. Enrofloxacin (Enro) was loaded into the KGM/PVDF hybrid microfibers, and the Enro release performance was tested. The sample showed excellent sustained release performance and applications in wound dressing.

KGM enhancement (Fig. $2 \mathrm{~b}$ and c). When the ratio of KGM : PVDF was $1: 10(\mathrm{w} / \mathrm{w})$, the surfaces of the microfibers were smooth and crack-free (Fig. 2d). The diameter of the microfibers decreased with an increase in the flow rate. The microfiber with the smallest diameter was obtained with an average diameter of $824.7 \pm 0.3 \mathrm{~nm}$ (Fig. 2d) under the conditions of $500 \mathrm{rad} \mathrm{min}^{-1}$ rotation and $80 \mathrm{~Hz}$ forward step. The core flow and sheath flow were fixed at $3 \mathrm{~mL} \mathrm{~h}^{-1}$ and $9 \mathrm{~mL} \mathrm{~h}^{-1}$, respectively.

\subsection{The characterization of hybrid microfibers}

The IR spectra of the microfibers in the range from 4000 to $400 \mathrm{~cm}^{-1}$ are shown in Fig. 3a. KGM contains many hydroxyl groups, allowing it to be highly hydrophilic, and it can easily form hydrogen bonds. ${ }^{40}$ PVDF contains many C-F groups, allowing the segments to have hydrophobic properties. ${ }^{41}$ The absorption band near $3416 \mathrm{~cm}^{-1}$ represents the stretching vibration of $\mathrm{O}-\mathrm{H}$ in $\mathrm{KGM} ;^{40}$ the peak at $1252 \mathrm{~cm}^{-1}$ is due to the $\mathrm{C}-\mathrm{N}$ stretching in Enro. ${ }^{42}$ The characteristic stretching vibrations noticed at $1172 \mathrm{~cm}^{-1}$ are due to $\mathrm{C}-\mathrm{F}$ in PVDF. ${ }^{41}$ The KGM/ PVDF or KGM/PVDF/Enro hybrid microfiber stretching frequencies shift to lower wavelengths at absorption band near
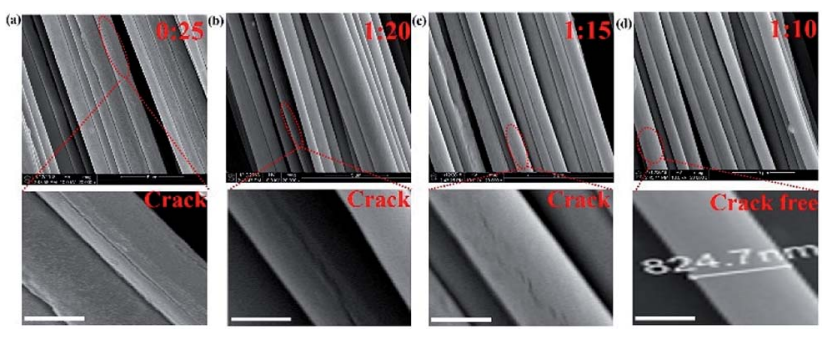

Fig. 2 (a) The SEM images of hybrid microfibers prepared with different weight ratios of KGM : PVDF: (a) $0: 25$, (b) $1: 20$, (c) $1: 15$, and (d) $1: 10$. Scale bars: $500 \mathrm{~nm}$. 

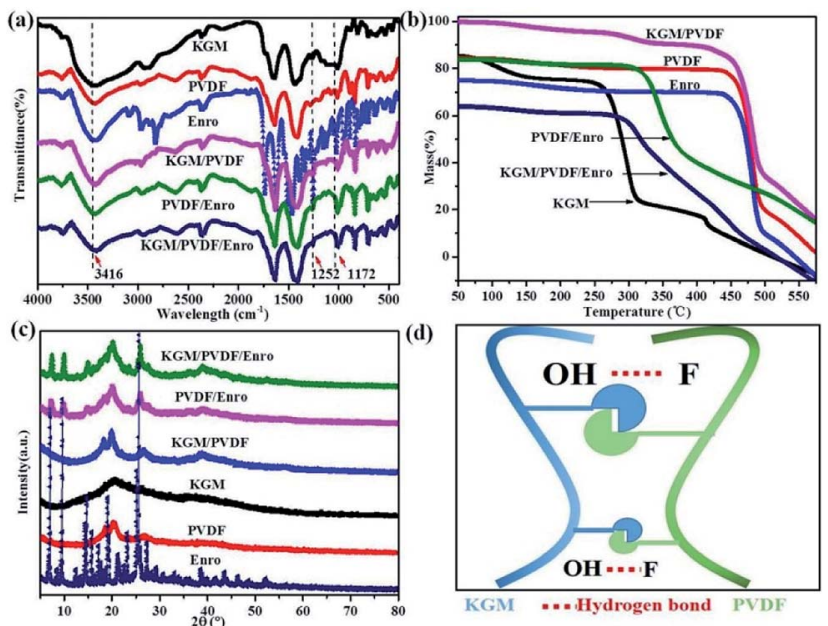

Fig. 3 (a) IR spectra of hybrid microfibers, (b) TGA patterns of hybrid microfibers, (c) XRD patterns of hybrid microfibers, and (d) hydrogen bond schematic between KGM and PVDF.

$3416 \mathrm{~cm}^{-1}$, and the stretching vibrations become weaker at the absorption band at $1172 \mathrm{~cm}^{-1}$ versus KGM or PVDF (Fig. 3a), which indicates that many hydrogen bonds are formed between $\mathrm{O}-\mathrm{H}$ and $\mathrm{C}-\mathrm{F}$ (Fig. 3d). The strong hydrogen bonding strengthens the attraction between the molecules in the microfibers, and the cracks on the surface of the microfibers disappear. TGA details the thermal property of the microfibers (Fig. 3b). The weight of KGM remains unchanged until the temperature rises to $250{ }^{\circ} \mathrm{C}$, at which the second stage begins. At this stage, the weight loss rate drops rapidly, and the sample loses $55 \%$ of its weight up to $325{ }^{\circ} \mathrm{C}$. The weight of PVDF changes from $430{ }^{\circ} \mathrm{C}$ to $490{ }^{\circ} \mathrm{C}$, and it loses $65 \%$ of its weight. However, in the case of $\mathrm{KGM} / \mathrm{PVDF}$, the situation is slightly different. The degradation in the second stage takes place at $460{ }^{\circ} \mathrm{C}$, followed by a relatively gradual decline in weight until $500{ }^{\circ} \mathrm{C}$. Unlike the results for KGM or PVDF, the temperature of $\mathrm{KGM} / \mathrm{PVDF}$ at which decomposition begins in the second stage is higher. Furthermore, $45 \%$ of the total weight of KGM/PVDF is lost in this stage, whereas only about $55 \%$ or $65 \%$ of the weight of the counterpart is lost in the case of KGM or PVDF; this indicates that the hydrogen bond between KGM and PVDF provides powerful thermal stability. Similarly, there is a sharp decline in PVDF/Enro versus KGM/PVDF/Enro, which indicates that the KGM/PVDF/Enro hybrid microfibers are more thermally stable. The XRD patterns of microfibers are shown in Fig. 3c. It is shown that the strong peaks at $2 \theta$ that correspond to $20^{\circ}$ and $25^{\circ}$ are assigned to PVDF. ${ }^{43}$ No new visible signal is observed for the KGM/PVDF hybrid microfibers. For a hybrid containing KGM, no difference is found in the X-ray patterns.

\subsection{Drug release in vitro}

We investigated the drug release performance by IR imaging and Korsmeyer-Peppas model. The maximum absorption wave and calibration curve of Enro are shown in Fig. S1 and S2. $\dagger$ The hybrid microfibers exhibited a burst release of Enro during the first $15 \mathrm{~h}$ followed by a sustained released for 13 days (Fig. 4a
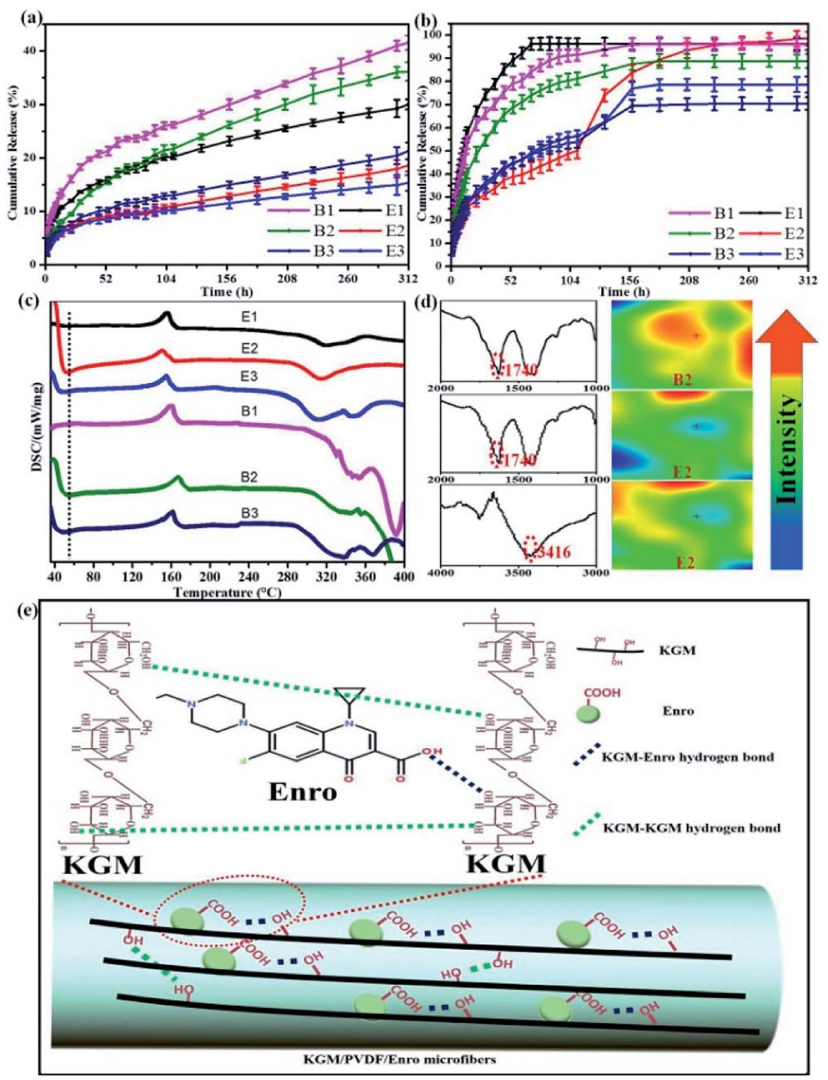

Fig. 4 (a) Cumulative release curves at $25^{\circ} \mathrm{C}$, (b) Cumulative release curves at $37{ }^{\circ} \mathrm{C}$, and (c) DSC curves of the microfibers. (d) The IR spectra and IR images illustrate the distribution of the carboxyl groups and hydroxyl groups before and after KGM addition of microfibers. (e) Schematic representation of sustained drug release via hydrogen bonding.

and $\mathrm{b}$ ). This phenomenon indicated that the hybrid microfibers are quite suitable for wound healing, which is a timeconsuming process. Compared with others, E2 showed significantly higher $(p<0.05)$ EEs and DLEs (Table 1$)$. Therefore, B2 and E2 as representatives were characterized by IR spectra and IR images to investigate the role of the functional groups (Fig. 4d). IR images show chemical changes from KGM addition and directly illustrate the distribution of carboxyl groups and hydroxyl groups through color variation (red represents high intensity and blue is low intensity). The uniform green color on the microfiber surface suggested that the carboxyl groups and hydroxyl groups were dispersed equally. ${ }^{\mathbf{4 4 , 4 5}}$ B2 had a large number of dangling carboxyl groups $\left(1740 \mathrm{~cm}^{-1}\right)$ from Enro; the carboxyl groups became relatively uniform on E2. Similarly, hydroxyl groups from KGM became relatively uniform $\left(3416 \mathrm{~cm}^{-1}\right.$ ) on $\mathrm{E} 2$. These results suggested that hydrogen bondings between the hydroxyl groups on KGM or between hydroxyl groups and carboxyl groups (KGM-Enro) were formed.

We further used the Korsmeyer-Peppas model ${ }^{\mathbf{4 6}}$ to investigate the kinetics of Enro release:

$$
\frac{M_{t}}{M_{\infty}}=K_{\mathrm{p}} t^{n}
$$


Table 1 The encapsulation efficiencies (EEs) and drug loading efficiencies (DLEs) of hybrid microfibers ${ }^{a}$

\begin{tabular}{|c|c|c|c|c|c|c|}
\hline Sample & E1 & E2 & E3 & B1 & B2 & B3 \\
\hline EEs (\%) & $96.22 \pm 2.48^{\mathrm{a}}$ & $98.5 \pm 2.99^{\mathrm{a}}$ & $78.59 \pm 3.45^{\mathrm{b}}$ & $96.1 \pm 2.66^{\mathrm{a}}$ & $88.7 \pm 2.99^{\mathrm{a}}$ & $70.46 \pm 2.60^{\mathrm{a}}$ \\
\hline DLEs (\%) & $8.718 \pm 0.03^{\mathrm{a}}$ & $13.4 \pm 0.04^{\mathrm{a}}$ & $13.37 \pm 0.06^{\mathrm{b}}$ & $9.5 \pm 0.02^{\mathrm{a}}$ & $13.08 \pm 0.04^{\mathrm{a}}$ & $13.29 \pm 0.03^{\mathrm{a}}$ \\
\hline
\end{tabular}

${ }^{a}$ Data are expressed as the mean $\pm \operatorname{SD}(n=3)$, different superscript letters in the same column mean that values are significantly different $(p<0.05)$.

Table 2 Results of Enro release curves fitting into Korsmeyer-Peppas model at $25^{\circ} \mathrm{C}$

\begin{tabular}{|c|c|c|}
\hline Samples & Equations & $R^{2}$ \\
\hline E1 & $\frac{M_{t}}{M_{\infty}}=4.48 t^{0.33}$ & 0.9932 \\
\hline E2 & $\frac{M_{t}}{M_{\infty}}=2.47 t^{0.34}$ & 0.9788 \\
\hline E3 & $\frac{M_{t}}{M_{\infty}}=2.63 t^{0.30}$ & 0.9887 \\
\hline B1 & $\frac{M_{t}}{M_{\infty}}=5.05 t^{0.36}$ & 0.9923 \\
\hline B2 & $\frac{M_{t}}{M_{\infty}}=2.60 t^{0.47}$ & 0.9970 \\
\hline B3 & $\frac{M_{t}}{M_{\infty}}=2.55 t^{0.36}$ & 0.9898 \\
\hline
\end{tabular}

here, $\frac{M_{t}}{M_{\text {}}}$ is the amount of drug release at time $t ; K_{\mathrm{p}}$ is the Peppas release rate constant that is related to the properties of the release system, and $n$ is the release exponent, which illustrates the transport mechanism.

The release exponent ( $n$ ) values for E1, E2, E3, B1, B2, and B3 at $25{ }^{\circ} \mathrm{C}$ were $0.33,0.34,0.30,0.36,0.47$, and 0.36 (Table 2), respectively; at $37^{\circ} \mathrm{C}$, the values were $0.23,0.40,0.38,0.26,0.50$, and 0.34 (Table 3), respectively, indicating that the main release mechanism is Fickian diffusion. ${ }^{47}$ The release exponent $(n)$ of E2 was less than that of B2, indicating that KGM's attraction forces for Enro reduced the speed of Fickian diffusion. The Peppas release rate constant $\left(K_{\mathrm{p}}\right)$ of $\mathrm{B} 2$ was higher than that of E2, which could be due to PVDF's repulsive forces for drugs by C-F groups. Single KGM microfibers have been reported to have disadvantages of fast dissolution due to their high hydrophilicity. ${ }^{23,25}$ Single PVDF microfibers have low drug-loading

Table 3 Results of Enro release curves fitting into Korsmeyer-Peppas model at $37^{\circ} \mathrm{C}$

\begin{tabular}{lll}
\hline Samples & Equations & $R^{2}$ \\
\hline E1 & $\frac{M_{t}}{M_{\infty}}=29.3 t^{0.23}$ & 0.8904 \\
E2 & $\frac{M_{t}}{M_{\infty}}=5.88 t^{0.40}$ & 0.9651 \\
E3 & $\frac{M_{t}}{M_{\infty}}=9.74 t^{0.38}$ & 0.9855 \\
B1 & $\frac{M_{t}}{M_{\infty}}=24.21 t^{0.26}$ & 0.9299 \\
B2 & $\frac{M_{t}}{M_{\infty}}=18.06 t^{0.50}$ & 0.9472 \\
B3 & $\frac{M_{t}}{M_{\infty}}=11.07 t^{0.34}$ & 0.9839
\end{tabular}

capacity as a result of high hydrophobicity. ${ }^{43,48}$ In general, KGM enhanced the drug-loading capacity of the microfibers based on the strong hydrogen bonds formed between the hydroxyl groups on KGM or between hydroxyl groups and carboxyl groups (KGM-Enro); by using PVDF, we could solve the fast dissolution problem of KGM based on the hydrophobic C-F groups. The balance between hydrophilic KGM and hydrophobic PVDF enabled the microfibers to achieve sustained drug release for 13 days.

Interestingly, more amounts of drug were released at $37{ }^{\circ} \mathrm{C}$ compared with that at $25{ }^{\circ} \mathrm{C}$ under the same conditions. By comparison, the $\mathrm{CR}$ values of E2 were $18.64 \pm 1.14 \%$ at $25{ }^{\circ} \mathrm{C}$ and $98 \pm 2.99 \%$ at $37{ }^{\circ} \mathrm{C}$ at $312 \mathrm{~h}$ (13 days). Similarly, the DSC curves exhibited fluctuation at low temperature (Fig. 4c), which suggested that the release performance of hybrid microfibers was temperature-sensitive. According to the literature, the amount of drug release increases with the increasing temperature, ${ }^{49}$ but such a large gap cannot be caused only by temperature. This was also due to the interactions between the hydrophilic KGM and hydrophobic PVDF, which can be beneficial for drug-loaded microfibers that will be stored for a long period of time.

\subsection{Test of antibacterial activity}

In vitro experiments were performed with $E$. coli and $S$. aureus to evaluate the antibacterial effects of the samples. The samples exhibited similar antibacterial effects against $E$. coli and $S$. aureus (Fig. 5). Clear zones surrounding B2 and E2 are shown in Fig. 5a. However, the antibacterial performance of B2 was slightly inferior to that of E2 due to its initial burst release, which indicated that E2 had better sustained drug release performance. It is well-known that a drug can cause waste and produce toxic side effects upon fast release. An extreme slow release results in low efficiency germicidal activity. ${ }^{51}$

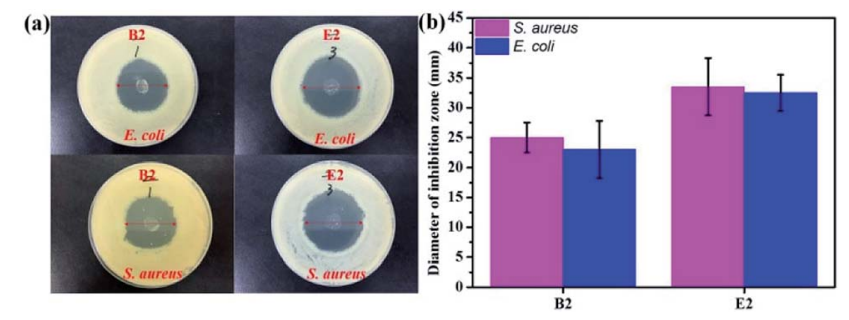

Fig. 5 Antibacterial activities evaluated by the zone of inhibition assay against E. coli and S. aureus; (a) photos of inhibition zone, (b) diameter of inhibition zone. 
Antibacterial experiments showed that the KGM/PVDF/Enro hybrid microfibers had a continuous bactericidal effect. Meanwhile, KGM played a supporting role in the sterilization of drugs. The high antibacterial activity of E2 suggested better wound-healing capability. ${ }^{50}$

\subsection{Applications in mice wound healing}

Finally, we used these microfibers as wound dressings for in vivo wound healing experiments. The drug release and antibacterial activity experiments described above showed that the hybrid microfibers possessed excellent sustained drug release performance and antibacterial properties. We assessed the degree of healing in terms of wound size. Fig. $6 \mathrm{~b}$ and $d$ show the wound closure macroscopic images and quantification analysis of the healing process of different test groups on the closure rate. It is clear that the hair follicles and neovascularization were observed, and the wound area gradually reduced. In contrast to the ones that were gauze-covered, fewer hair follicles and neovascularization were observed for the wounds covered with the PVDF/Enro microfibers. Significantly, for the wounds treated with the KGM/PVDF/Enro hybrid microfibers, more neovascularization and mature hair follicles were observed in the regenerated epidermis at the

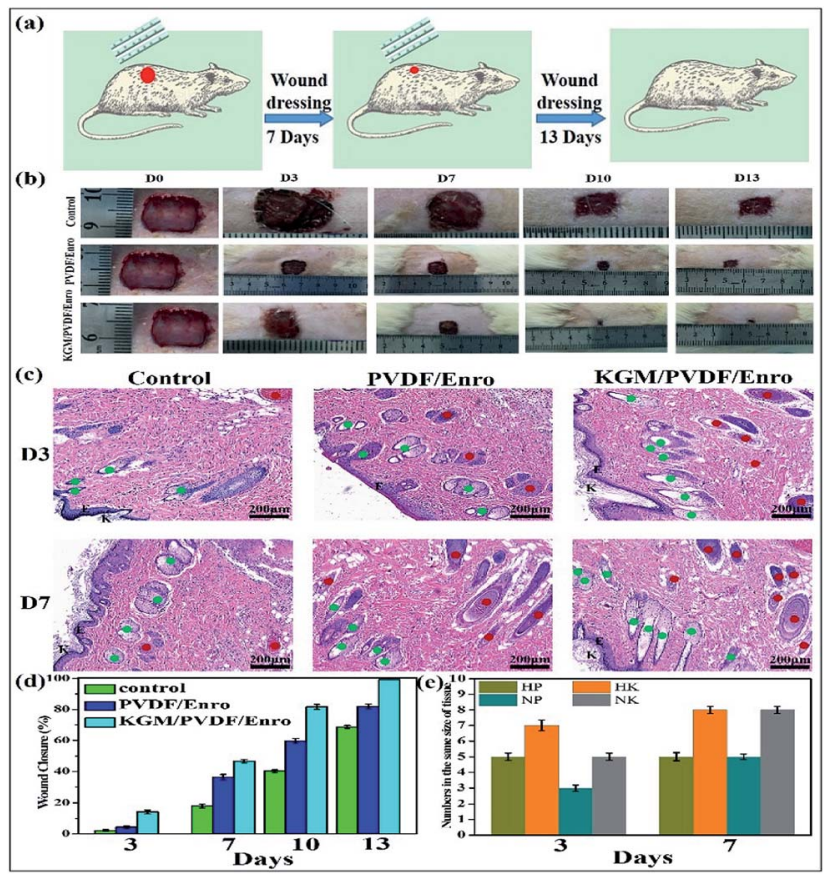

Fig. 6 Effects of PVDF/Enro, KGM/PVDF/Enro microfibers on wound healing, control group (gauze covered) used for comparison. (a) Schematic representation of the healing wound, (b) photographic evaluations of the healing process, (c) histology of wounds at 3 days and 7 days. K: keratin layer, Epi: epidermis, N: neovascularization ( $\bullet$ ), F: hair follicles ( $\bullet$ ), (d) ratio of wound closure during wound healing, (e) numbers in the same size of tissue. HP: keratin layer of PVDF/Enro microfibers, HK: keratin layer of KGM/PVDF/Enro hybrid microfibers, NP: neovascularization of PVDF/Enro microfibers, NK: neovascularization of KGM/PVDF/Enro hybrid microfibers. same size of the tissue (Fig. 6c and e). The wounds covered with the KGM/PVDF/Enro hybrid microfibers also showed a faster healing process than the observations for the other two groups. Particularly, by day $13,99.3 \pm 0.1 \%$ of the injured area had reepithelialized after treatment with KGM/PVDF/Enro hybrid microfibers. In contrast, only $82 \pm 1.48 \%$ and $68.7 \pm$ $0.96 \%$ of the wound areas had healed when PVDF/Enro microfibers and gauze, respectively, were used (Fig. 6d). This can be ascribed to the hybrid microfibers' sustained drug release for 13 days based on the balance between hydrophilic KGM and hydrophobic PVDF, which reduced the repeated pain caused during the replacement of wound dressings. ${ }^{52-54}$ The results further demonstrated that the KGM/PVDF hybrid microfibers have excellent sustained drug release performance. Particularly, these hybrid microfibers having hydrophilic and hydrophobic segments produced via the microfluidic spinning concept can promote wound healing and tissue regeneration.

\section{Conclusions}

In summary, we report a simple and rapid strategy for the in situ preparation of KGM/PVDF hybrid microfibers as a novel wound dressing via microfluidic spinning. The interaction between hydrophilic KGM and hydrophobic PVDF resulted in hybrid microfibers that exhibited crack-free surface, excellent heat resistance and sustained drug release performance. More specifically, the hydrogen bonds formed between $\mathrm{O}-\mathrm{H}$ of the hydrophilic KGM and C-F of the hydrophobic PVDF resulted in hybrid microfibers having crack-free surfaces and excellent heat resistance; the balance between KGM's attraction forces, which are produced by the hydrogen bonds (KGM-KGM or KGM-Enro), and PVDF's repulsive forces, which are formed due to the existence of $\mathrm{C}-\mathrm{F}$ groups, promoted sustained drug release for 13 days. This wound dressing has excellent antibacterial activity against $E$. coli and $S$. aureus and can reduce repeated pain caused by the replacement of a wound dressing. A new hydrophilic/hydrophobic drug carrier structure has been designed, which has solved the fast dissolution problem of KGM and the low drug-loading capacity demerit of PVDF; it can also reduce the possibility of generating cracks in the field of micromanipulation. This tool is potentially useful for various applications in biomaterials.

\section{Conflicts of interest}

There are no conflicts to declare.

\section{Acknowledgements}

This work was supported by the National Natural Science Foundation of China $(31772045,31471704)$. The authors thank Janus New-Materials Co., Ltd. for assistance with microfluidic spinning technique. The authors thank Qing Li for valuable discussion on preparation of the experiment. 


\section{Notes and references}

1 N. C. Tansil, L. D. Koh and M. Y. Han, Adv. Mater., 2012, 24, 1388-1397.

2 S. Zhao, L. Li, H. Wang, Y. Zhang, X. Cheng, N. Zhou, M. N. Rahaman, Z. Liu, W. Huang and C. Zhang, Biomaterials, 2015, 53, 379-391.

3 P. Martin, Science, 1997, 276, 75-81.

4 A. H. Najafabadi, A. Tamayol, N. Annabi, M. Ochoa, P. Mostafalu, M. Akbari, M. Nikkhah, R. Rahimi, M. R. Dokmeci, S. Sonkusale, B. Ziaie and A. Khademhosseini, Adv. Mater., 2014, 26, 5823-5830.

5 I. Liakos, L. Rizzello, H. Hajiali, V. Brunetti, R. Carzino, P. P. Pompa, A. Athanassiou and E. Mele, J. Mater. Chem. B, 2015, 3, 1583-1589.

6 N. Elkhatib, E. Bresteau, F. Baschieri, A. L. Rioja, G. V. Niel, S. Vassilopoulos and G. Montagnac, Science, 2017, 356, 1-8.

7 X. Zhao, H. Wu, B. Guo, R. Dong, Y. Qiu and P. X. Ma, Biomaterials, 2017, 122, 34-47.

8 C. Zhong, T. Gurry, A. A. Cheng, J. Downey, Z. Deng, C. M. Stultz and T. K. Lu, Nat. Nanotechnol., 2014, 9, 858-866.

9 A. Q. Chen, H. C. He, G. L. Ma, Y. Li, S. S. Jiang, X. Xuan, Y. Song, C. Y. Zhang, J. Xiao, Y. S. Xu, J. Wu and S. F. Chen, RSC Adv., 2018, 8, 10620-10626.

10 A. Khalid, H. Ullah, M. Ul-Islam, R. Khan, S. Khan, F. Ahmad, T. Khan and F. Wahid, RSC Adv., 2017, 7, 47662-47668.

11 S. Shabahang, G. Tao, J. J. Kaufman, Y. Qiao, L. Wei, T. Bouchenot, A. P. Gordon, Y. Fink, Y. Bai, R. S. Hoy and A. F. Abouraddy, Nature, 2016, 534, 529-533.

12 A. T. Haedler, K. Kreger, A. Issac, B. Wittmann, M. Kivala, N. Hammer, J. Kohler, H. W. Schmidt and R. Hildner, Nature, 2015, 523, 196-199.

13 A. Bloessera, P. Voepela, M. O. Loeha, A. Beyerb, K. Volzb and R. Marschalla, J. Mater. Chem. A, 2018, 6, 1971-1978.

14 D. Psaltis, S. R. Quake and C. Yang, Nature, 2006, 442, 381386.

15 D. Li, A. Babel, S. A. Jenekhe and Y. Xia, Adv. Mater., 2004, 16, 2062-2066.

16 G. F. J. Müller, M. Stürzel and R. Mülhaupt, Adv. Funct. Mater., 2014, 24, 2860-2864.

17 R. J. DeVolder, H. Bae, J. Lee and H. Kong, Adv. Mater., 2011, 23, 3139-3143.

18 G. M. Whitesides, Nature, 2006, 442, 368-373.

19 R. V. Bell, C. C. Parkins, R. A. Young, C. M. Preuss, M. M. Stevens and S. A. F. Bon, J. Mater. Chem. A, 2016, 4, 813-818.

20 M. Y. Jiang, X. J. Ju, K. Deng, X. X. Fan, X. H. He, F. Wu, F. He, Z. Liu, W. Wang, R. Xiea and L. Y. Chu, J. Mater. Chem. B, 2016, 4, 3925-3935.

21 E. K. Sackmann, A. L. Fulton and D. J. Beebe, Nature, 2014, 507, 181-189.

22 Y. Zhang, Y. Tian, L. L. Xu, C. F. Wang and S. Chen, Chem. Commun., 2015, 51, 17525-17528.

23 R. J. Mu, Y. Ni, L. Wang, Y. Yuan, Z. Yan, J. Pang and S. Chen, Mater. Lett., 2017, 196, 410-413.
24 Y. S. Zhang and A. Khademhosseini, Science, 2017, 356(6337), eaaf3627.

25 Y. Ni, R. J. Mu, X. D. Tan, R. X. Huang, Y. Yuan, H. B. Chen and J. Pang, Struct. Chem., 2017, 36, 1043-1048.

26 R. J. Mu, Y. Yuan, L. Wang, Y. Ni, M. Li, H. Chen and J. Pang, Food Hydrocolloids, 2018, 76, 42-48.

27 L. Wu, X. Lin, J. Wu, X. Zhou and X. Luo, RSC Adv., 2016, 6, 89417-89429.

28 L. Persano, C. Dagdeviren, Y. Su, Y. Zhang, S. Girardo, D. Pisignano, Y. Huang and J. A. Rogers, Nat. Commun., 2013, 4, 1633-1643.

29 Z. L. Luo, Y. Li, C. Duan and B. B. Wang, RSC Adv., 2018, 8, 16251-16259.

30 C. Wan and C. R. Bowen, J. Mater. Chem. A, 2017, 5, 30913128.

31 C. D. Ma, C. Wang, C. Acevedo-Velez, S. H. Gellman and N. L. Abbott, Nature, 2015, 517, 347-363.

32 T. He, J. Wang, P. Huang, B. Zeng, H. Li, Q. Cao, S. Zhang, Z. Luo, D. Y. Deng, H. Zhang and W. Zhou, Colloids Surf., $B, 2015,130,278-286$.

33 S. S. Behera and R. C. Ray, Int. J. Biol. Macromol., 2016, 92, 942-956.

34 Y. Yuan, L. Wang, R. J. Mu, J. N. Gong, Y. Wang, Y. Li, J. Ma, J. Pang and C. Wu, Carbohydr. Polym., 2018, 190, 196-203.

35 J. Tang, J. Mi, W. Huang, H. Zhong, Y. Li, J. Zhou and A. M. Johri, J. Mater. Chem. B, 2018, 6, 1920-1929.

36 Y. Lyu, H. Ren, M. Yu, X. Li, D. Li and C. Mu, Carbohydr. Polym., 2017, 174, 1095-1105.

37 D. Q. Wu, H. C. Cui, J. Zhu, X. H. Qin and T. Xie, J. Mater. Chem. B, 2016, 4, 2606-2613.

38 W. C. Lin, C. C. Lien, H. J. Yeh, C. M. Yu and S. H. Hsu, Carbohydr. Polym., 2013, 94, 603-611.

39 Q. Li, Y. Niu, H. Diao, L. Wang, X. Chen, Y. Wang, L. Dong and C. Wang, Biomaterials, 2017, 148, 54-68.

40 Z. Li, Y. Su, B. Xie, X. Liu, X. Gao and D. Wang, J. Mater. Chem. B, 2015, 3, 1769-1778.

41 J. Lv, G. Zhang, H. Zhang, C. Zhao and F. Yang, Appl. Surf. Sci., 2018, 440, 1091-1100.

42 L. Zhang, D. Dong, X. Hua and Z. Guo, Sci. Total Environ., 2018, 625, 178-184.

43 B. Luo, X. Wang, Y. Wang and L. Li, J. Mater. Chem. A, 2014, 2, 510-519.

44 C. Xu, Q. Li, X. T. Hu, C. F. Wang and S. Chen, J. Mater. Chem. $B, 2017$, 5, 3816-3822.

45 C. Yu, C. F. Wang and S. Chen, J. Mater. Chem. A, 2015, 3, 17351-17358.

46 A. Tamayo, M. A. Mazo, M. D. Veiga, R. Ruiz-Caro, F. NotarioPerez and J. Rubio, Mater. Sci. Eng., C, 2017, 75, 1097-1105.

47 J. D. Obayemi, Y. Danyuo, S. Dozie-Nwachukwu, O. S. Odusanya, N. Anuku, K. Malatesta, W. Yu, K. E. Uhrich and W. O. Soboyejo, Mater. Sci. Eng., C, 2016, 66, 51-65.

48 D. Li, Y. Wang, J. Hu, B. Lu, D. Dang, J. Zhang and Y. T. Cheng, J. Power Sources, 2018, 387, 9-15.

49 Y. Xu, J. J. Li, D. J. Yu, G. R. Williams, J. H. Yang and X. Wang, Eur. J. Pharm. Sci., 2017, 106, 422-430. 
50 D. Simoes, S. P. Miguel, M. P. Ribeiro, P. Coutinho, A. G. Mendonca and I. J. Correia, Eur. J. Pharm. Biopharm., 2018, 127, 130-141.

51 D. Mishraa, P. Kharea, D. K. Singhb, S. Luqmanb, P. V. A. Kumarc, A. Yadavc, T. Dasd and B. K. Saikiad, Ind. Crops Prod., 2018, 114, 68-80.
52 M. Naseri-Nosar and Z. M. Ziora, Carbohydr. Polym., 2018, 189, 379-398.

53 S. F. Chou, D. Carson and K. A. Woodrow, J. Controlled Release, 2015, 220, 584-591.

54 E. Pinho and G. Soares, J. Mater. Chem. B, 2018, 6, 18871898. 